\title{
Nucleonic resonance effects in the $\phi$ meson photoproduction
}

\author{
Qiang Zhao $^{\text {a }}$, J.-P. Didelez ${ }^{\text {a }}$, M. Guidal ${ }^{\text {a }}$, B. Saghai ${ }^{\mathrm{b}}$ \\ ${ }^{a}$ Institut de Physique Nucléaire, F-91406 Orsay Cedex, France \\ b Service de Physique Nucléaire, DAPNIA-DSM, CEA/Saclay, \\ F-91191 Gif-sur-Yvette, France
}

\begin{abstract}
The process $\gamma p \rightarrow \phi p$ close to threshold is investigated focusing on the role played by the $s$ - and $u$-channel nucleonic resonances. For this purpose, a recent quark model approach, based on the $S U(6) \otimes O(3)$ symmetry with an effective Lagrangian, is extended to the $\phi$ meson photoproduction. Another non-diffractive process, the $t$ channel $\pi^{0}$ exchange, is also included. The diffractive contribution is produced by the $t$-channel Pomeron exchange. Contributions from non-diffractive $s$ - and $u$-channel process are found small in the case of cross sections and polarization observables at forward angles. However, backward angle polarization asymmetries show high sensitivity to this non-diffractive process. Different prescriptions to keep gauge invariance for the Pomeron exchange amplitudes are investigated. Possible deviations from the exact $S U(6) \otimes O(3)$ symmetry, due to the configuration mixing, are also discussed.
\end{abstract}

PACS: 12.39.-x, 25.20.Lj, 13.60.Le, 13.88

Keywords: Phenomenological quark model, Photoproduction reactions, Meson production, Polarization 


\section{Introduction}

A well established feature in vector meson photoproduction at low momentum transfer and high energies is that the diffractive scattering governs the reaction mechanism [1]. With the advent of the new high intensity beam facilities, like JLAB, ELSA, GRAAL, SPring-8, the study of this field in both low energy $\left(E_{\gamma} \sim 2 \mathrm{GeV}\right)$ and/or high momentum transfer $\left(-t \geq 1(\mathrm{GeV} / \mathrm{c})^{2}\right)$ becomes possible. In these regimes, deviations from pure diffractive phenomena are expected, which should show up, especially in polarization observables.

In recent works, the diffractive component is dealt with as a $t$-channel Pomeron exchange [2-5], where the Pomeron is treated as a $C=+1$ isoscalar photon. For the non-diffractive contributions, various sources have been explored. In Ref. [6], the $t$-channel pseudoscalar mesons $\left(J^{\pi}=0^{-}\right.$, i.e. $\pi^{0}$ and $\left.\eta\right)$ exchanges are in general found to produce small contributions at small momentum transfers. Here, it will be interesting to study the nature of the parity exchange at low energy, namely, the interplay between diffractive scattering which is intrinsically a natural parity exchange and the pseudoscalar meson exchange which is an unnatural parity exchange process.

Another exciting topic concerns the possible violation mechanisms of the Okubo-Zweig-Iizuka (OZI) rule [7]. Such a violation has recently been reported in the $\bar{p} n$ and $p \bar{p}$ annihilation experiments [8]. Theoretically, these phenomena can not be explained by conventional approaches based on the two-step OZI allowed final state interaction with the intermediate kaon formation. Various models have been proposed to account for this, initiating the search for strangeness components in nucleons via the $\phi$ meson photoproduction near threshold [9-11]. Then, one needs to investigate on the one hand OZI evading processes and on the other hand the role of the strangeness content of the proton via $s \bar{s}$ knock-out reactions.

Another important component in the reaction mechanism at low energies is expected to be the contributions from $s$ - and $u$-channel resonance excitations. At present time, there is no systematic investigation of the role played by the resonances in the $\phi$ meson photoproduction, which, however, might be another important non-diffractive source contributing near threshold. Although a recent work [12] taking into account the $s$ - and $u$-channel $\phi N N$ coupling has been carried out, the contributions from resonances have not been included there. In fact, at hadronic level, the unknown $\phi N N^{*}$ couplings have been the barrier to go further to include the resonances since one has to introduce at least one parameter for each $\phi N N^{*}$ coupling vertex, therefore, a large number of parameters will appear in the theory. On this point, the quark model approach shows great advantages: in the exact $S U(6) \otimes O(3)$ symmetry limit, the quark-vector-meson interactions can be described by the effective Lagrangian 
with only two parameters. The main purpose of the present work is, therefore, to focus on this aspect using a quark-model based effective Lagrangian approach in line with a recent work $[13,14]$. Here, we wish to emphasize that in the latter work, a very preliminary study of the resonance contributions to the $\phi$ meson photoproduction was performed and, especially, the dominant diffractive contribution was not included. Therefore, it was impossible to constrain the parameters there and an approximate estimation was made for only the differential cross section from the resonance contributions. In this work, the dominant diffractive process has been introduced consistently with the quark model approach, and a search is performed to constraint the parameters. This set of ingredients is expected to make possible a reliable estimation of the small, but significant, contributions from the nucleonic resonances to the reaction mechanism. Our results show that the resonances (with mass $M_{R} \leq$ $2.0 \mathrm{GeV}$ ) play a non-negligible role, especially in polarization observables, in spite of the rather high $\phi$ meson production threshold $\left(E_{\gamma}^{\text {thres }} \sim 1.57 \mathrm{GeV}\right.$ corresponding to the total c.m. energy $\sqrt{s} \sim 1.96 \mathrm{GeV})$.

As a first step of study in the energy region near threshold, we employ a nonrelativistic quark potential to describe the quark motion. As it has been shown in Ref. [15], the non-relativistic formulation remains a viable approximation due mainly to the effective parameters such as the constituent quark mass. To partly cure the shortcoming arising from the non-relativistic quark potential, a widely used Lorentz boost is introduced in the spatial integrals [16].

For the pure diffractive process, our formalism embodies a model treating the Pomeron like a $C=+1$ isoscalar photon $[2,5]$. It is the extrapolation from the high energy region $\left(E_{\gamma}=6.45 \mathrm{GeV}\right)$ down to the low energy region. Thus, no free parameters are introduced. The resonance contributions drop quickly with the energy increasing, and almost vanish when the photon energy goes above roughly $2.8 \mathrm{GeV}$. So, at higher energies, the $\phi$ meson photoproduction cross section is generated by the diffractive process. Therefore, with such a Pomeron exchange term, this model can be applied to the $\phi$ meson production from threshold up to the higher energy region (i.e. $E_{\gamma} \sim 10 \mathrm{GeV}$ ) where the $s$ and $u$-channel contributions are negligible. Although our interest is to study the energy region near threshold, we emphasize that a reliable estimation of the diffractive contribution in this energy region must be the prerequisite to further investigations, especially, when focusing on the small non-diffractive contributions.

The contribution from $\pi^{0}$ exchange is also included in this work. However, we find that at $E_{\gamma}=2.0 \mathrm{GeV}$, it is quite negligible in comparison with not only the Pomeron exchange in the small $|t|$ region, but also the resonance contributions in the large $|t|$ region. We do not include the amplitude for the $\eta$ exchange in this work, since although the $\phi$ meson has larger decay branching ratio for $\phi \rightarrow \eta \gamma$ than for $\phi \rightarrow \pi^{0} \gamma$ which means that the $\phi \eta \gamma$ coupling is stronger than 
the $\phi \pi^{0} \gamma$ coupling, a recent analysis [17] of the $\eta$ photoproduction shows that $g_{\eta N N}$ is smaller than $g_{\pi N N}$ by roughly a factor of 7 , which leads to negligible effects due to the $\eta$ exchange in the $\phi$ production.

In section 2, we present the effective Lagrangian for the quark-vector-meson coupling and the amplitude for the Pomeron and $\pi^{0}$ exchanges. In section 3 , the numerical results for the differential cross section and polarization observables are reported. As a test of the model, we present the total cross section up to $E_{\gamma}=10 \mathrm{GeV}$ in comparison with the experimental data. We also present comparisons, for the three density matrix elements $\rho_{00}^{0}, \operatorname{Re} \rho_{10}^{0}$ and $\rho_{1-1}^{0}$, between our predictions and the experimental results, showing that the diffractive contribution has been treated in a reliable manner. The predictions for the three density matrix elements are presented at $E_{\gamma}=2.0 \mathrm{GeV}$. Possible OZI suppression effects are also discussed. In section 4, Pomeron exchange amplitudes, due to different schemes of taking into account the gauge invariance, are investigated. Conclusions are given in section 5 . 


\section{Formalism}

In Ref. [14], the quark model approach to vector meson photoproduction with an effective Lagrangian in the resonance region has been developed. In the following Subsection we summarize the main points of that approach and extend it to the $\phi$ meson photoproduction process. Notice that in Ref. [14] the latter process was investigated only qualitatively, for the following reasons: i) the diffractive Pomeron exchange, which plays a dominant role in the reaction mechanism, is absent in that work, ii) this latter shortcoming prevents any parameter search, iii) also, the polarization observables can not be investigated within Ref. [14] formalism.

In the remaining two subsections, the diffractive Pomeron exchange and the non-diffractive $\pi^{0}$ exchange are presented.

\subsection{Effective Lagrangian for quark-vector-meson coupling}

The formalism used here is based on the $S U(6) \otimes O(3)$ symmetry for the 3 -quark baryon system. We suppose that the $\phi$ meson is produced from an elementary process described by an effective Lagrangian where the $\phi$ meson is treated as a point-like elementary particle. As we will discuss later, deviations from this symmetry are a valuable source of information about the structure of the intervening hadrons.

The effective Lagrangian introduced for the quark-meson coupling has the following form:

$$
L_{e f f}=-\bar{\psi} \gamma_{\mu} p^{\mu} \psi+\bar{\psi} \gamma_{\mu} e_{q} A^{\mu} \psi+\bar{\psi}\left(a \gamma_{\mu}+\frac{i b \sigma_{\mu \nu} q^{\nu}}{2 m_{q}}\right) \phi_{m}^{\mu} \psi
$$

where $\psi$ and $\bar{\psi}$ represent the quark and anti-quark fields, respectively, and $\phi_{m}^{\mu}$ denotes the vector meson field. The two parameters, $a$ and $b$ represent the vector and tensor couplings of the quark to the vector meson, respectively, and $m_{q}=330 \mathrm{MeV}$ is the constituent quark mass.

With the above effective Lagrangian, at tree level, the non-diffractive transition amplitudes can be expressed as the sum of the contributions from $s^{-}, u^{-}$, and t-channel:

$$
M_{f i}=M_{f i}^{s}+M_{f i}^{u}+M_{f i}^{t} .
$$


Given that the $t$-channel contribution, $M_{f i}^{t}$, is proportional to the charge of the final state meson, it does not contribute in the case of neutral $\phi$ meson photoproduction. The transition amplitudes from $s$ - and $u$-channel can be written as:

$$
\begin{aligned}
M_{f i}^{s+u}= & i \omega_{\gamma} \sum_{j}\left\langle N_{f}\left|H_{m}\right| N_{j}\right\rangle\left\langle N_{j}\left|\frac{1}{E_{i}+\omega_{\gamma}-E_{j}} h_{e}\right| N_{i}\right\rangle \\
& +i \omega_{\gamma} \sum_{j}\left\langle N_{f}\left|h_{e} \frac{1}{E_{i}-\omega_{\phi}-E_{j}}\right| N_{j}\right\rangle\left\langle N_{j}\left|H_{m}\right| N_{i}\right\rangle,
\end{aligned}
$$

with $H_{m}=-\bar{\psi}\left(a \gamma_{\mu}+\frac{i b \sigma_{\mu \nu} q^{\nu}}{2 m_{q}}\right) \phi_{m}^{\mu} \psi$ for the quark-meson coupling vertex, and

$$
h_{e}=\sum_{l} e_{l} \mathbf{r}_{l} \cdot \boldsymbol{\epsilon}_{\gamma}(1-\boldsymbol{\alpha} \cdot \hat{\mathbf{k}}) e^{i \mathbf{k} \cdot \mathbf{r}_{l}}, \hat{\mathbf{k}}=\frac{\mathbf{k}}{\omega_{\gamma}} .
$$

Here, $l=1,2,3$, denotes the three quarks of the initial or final state nucleons, and $e_{l}$ is the charge of the $l$ th quark. The matrix, $\boldsymbol{\alpha}=\gamma^{0} \boldsymbol{\gamma}$, where $\gamma^{0}$ and $\gamma$ are the Dirac matrices. The kinematic variables are, $\mathbf{k}$ : the momentum of the incident photon; $\omega_{\gamma}$ : the photon energy; $\omega_{\phi}$ : the energy of the outgoing meson; $E_{j}$ : the energy of the intermediate state in the $s$ - and $u$-channels; $E_{i}$ : the energy of the initial state nucleon. It should be noted that in Eq. (3), we have omitted the contact term derived from the $s$ - and $u$-channels since it is proportional to the charge of the outgoing meson, therefore, vanishes in the neutral meson production processes.

The explicit expressions for the longitudinal and transverse $s$ - and $u$-channel transition matrices have been derived in Ref. [14]. The transition amplitudes for each resonance in the $s$-channel below $2 \mathrm{GeV}$ are included explicitly, while the resonances above $2 \mathrm{GeV}$ with a given quantum number $n>2$ in the harmonic oscillator basis of the quark model are treated as degenerate. The contributions from the $u$-channel resonances are divided into two parts as well. The first part contains the baryons with the quantum number $n=0$, which includes the spin $1 / 2$ states, and the spin $3 / 2$ resonances. Since the mass splitting between the spin $1 / 2$ and spin $3 / 2$ resonances with $n=0$ is significant, they have to be treated separately. The second part in the $u$-channel comes from the excited resonances with quantum number $n \geq 1$. As the contributions from the $u$-channel resonances are not sensitive to the precise mass positions, they can also be treated as degenerate. In the $\phi$ meson photoproduction, because of the isospin conservation, the $\phi$ meson photoproduction gets contributions only from isospin $1 / 2$ resonances. Therefore, only the nucleon pole term $(n=0)$ and those intermediate excited states $(n>0)$ with isospin 1/2 contribute in this reaction. Also, the Moorhouse selection rule [18] suppresses those states belonging to representation $\left(\mathbf{7 0},{ }^{\mathbf{4}} \mathbf{8}\right)$ from contributing in the photon excitations of the proton target. Therefore, in the NRCQM 
symmetry limit, there are only 8 intermediate nucleonic resonances appearing in the $s$-channel with $n \leq 2$. In Table 1 , the NRCQM wave-functions of these resonances are presented with their masses $M_{R}$ and total widths $\Gamma_{T}$.

The transition amplitudes can then be expressed in terms of the 12 independent helicity amplitudes, which are related to the spin observables and the density matrix elements $[19,20]$.

The general transition amplitude for the $s$-channel excited states in the helicity space has the following form:

$$
H_{a \lambda_{V}}^{J}=\frac{2 M_{R}}{s-M_{R}\left(M_{R}-i \Gamma(\mathbf{q})\right)} h_{a \lambda_{V}}^{J}
$$

where $\sqrt{s}=E_{i}+\omega_{\gamma}=E_{f}+\omega_{m}$ is the total energy of the system, $E_{i}$ and $E_{f}$ are the energies of the nucleons in the initial and final states, respectively, $h_{a \lambda_{V}}^{J}$ are the helicity amplitudes, and $\Gamma(\mathbf{q})$, which is a function of the final state momentum $\mathbf{q}$, denotes the momentum dependence of the total width of the resonance $\Gamma_{T}[16]$.

The differential cross section has the expression:

$$
\frac{\mathrm{d} \sigma}{\mathrm{d} t}=\frac{\alpha_{e}\left(E_{f}+M_{N}\right)\left(E_{i}+M_{N}\right)}{16 s|\mathbf{k}|^{2}} \frac{1}{2} \sum_{a=1}^{4} \sum_{\lambda_{V}=0, \pm 1}\left|H_{a \lambda_{V}}\right|^{2}
$$

Table 1

Resonances in the $s$-channel, with their assignments in the $S U(6) \otimes O(3)$ symmetry limit, are given in the first and second columns, respectively. The masses $\left(M_{R}\right)$ and and total widths $\left(\Gamma_{T}\right)$, used in this work, given in columns third and fourth, respectively, are taken from Ref. [21].

\begin{tabular}{cccc}
\hline Resonances & $S U(6) \otimes O(3)$ & $M_{R}(\mathrm{MeV})$ & $\Gamma_{T}(\mathrm{MeV})$ \\
\hline$P_{11}(1440)$ & $N\left({ }^{2} S_{S}^{\prime}\right)_{\frac{1}{2}}{ }^{+}$ & 1440 & 350 \\
$D_{13}(1520)$ & $N\left({ }^{2} P_{M}\right)_{\frac{3}{2}^{-}}$ & 1520 & 120 \\
$S_{11}(1535)$ & $N\left({ }^{2} P_{M}\right)_{\frac{1}{2}^{-}}$ & 1535 & 150 \\
$F_{15}(1680)$ & $N\left({ }^{2} D_{S}\right)_{\frac{5}{2}}^{+}$ & 1680 & 130 \\
$P_{11}(1710)$ & $N\left({ }^{2} S_{M}\right)_{\frac{1}{2}}^{+}$ & 1710 & 100 \\
$P_{13}(1720)$ & $N\left({ }^{2} D_{S}\right)_{\frac{3}{2}}^{+}$ & 1720 & 150 \\
$P_{13}(1900)$ & $N\left({ }^{2} D_{M}\right)_{\frac{3}{2}}^{+}$ & 1900 & 400 \\
$F_{15}(2000)$ & $N\left({ }^{2} D_{M}\right)_{\frac{5}{2}}{ }^{+}$ & 2000 & 450 \\
\hline
\end{tabular}


where $M_{N}$ represents the mass of the nucleon, and $\mathbf{k}$ denotes the momentum of the incoming photon in the c.m. system.

In the harmonic oscillator basis, the factor $e^{-\frac{\mathbf{q}^{2}+\mathbf{k}^{2}}{6 \alpha^{2}}}$ comes from the integrations over the 3-quark baryon wave-functions, and plays a role like a form factor for the quark-meson and quark-photon vertices [22]. Here, $\alpha=410 \mathrm{MeV}$ is the commonly used value for the harmonic oscillator strength, and no additional "cut-off" parameter is needed. Thus, only two parameters, $a$ and $b$ in Eq. (1) are introduced by the effective Lagrangian for the $s$ - and $u$-channel non-diffractive $\phi$ production. It should be noted that the gauge invariance of the amplitudes from the effective Lagrangian has been fixed. Moreover, the above factor is implemented with the Lorentz boost to take into account the relativistic effects.

\section{2 t-channel diffractive Pomeron exchange}

We use the Pomeron exchange model by Donnachie and Landshoff [2] to produce the diffractive contribution in this work. In the model, the Pomeron mediates the long range interaction between a confined quark and a nucleon. Although the nature of the Pomeron exchange is still unclear, it has been shown that the Pomeron exchange based on the Regge phenomenology is one of the most successful approaches to high energy elastic scattering. Also, it has been shown that the Pomeron behaves rather like a $C=+1$ isoscalar photon.

With the Pomeron-photon analogy picture, the Pomeron-nucleon coupling is described by the vertex:

$$
F_{\mu}(t)=3 \beta_{0} \gamma_{\mu} f(t)
$$

where $-t$ is the Pomeron momentum squared, $\beta_{0}$ gives the strength of the coupling of the single Pomeron to a light constituent quark. $f(t)$ represents the form factor which is taken to be the same as the isoscalar nucleon electromagnetic form factor, therefore it has the following expression:

$$
f(t)=F_{1}(t)=\frac{\left(4 M_{N}^{2}-2.8 t\right)}{\left(4 M_{N}^{2}-t\right)(1-t / 0.7)^{2}} .
$$

For the $\gamma \phi \mathcal{P}$ vertex, the lowest order diagram for the quark pair creation in Ref. [5] is used for the $s \bar{s}$ creation, but has been extrapolated to the limit of $Q^{2}=0$, namely, the process with real photons. A bare photon vertex is introduced for the quark-photon interaction, which has the same form as the 
quark-photon coupling in Eq. (1). Here, we use the "on-shell approximation" for the quark- $\phi$ vertex, i.e. $V_{\nu}$ in Fig. 1:

$$
V_{\nu}\left(p-\frac{1}{2} q, p+\frac{1}{2} q\right)=f_{\phi} M_{\phi} \gamma_{\nu}
$$

where $f_{\phi}$ represents the coupling strength and is fixed by the $\phi \rightarrow e^{+} e^{-}$decay width $\Gamma_{\phi} \rightarrow e^{+} e^{-}$with the following relation:

$$
\Gamma_{\phi \rightarrow e^{+} e^{-}}=\frac{8 \pi \alpha_{e}^{2} e_{Q}^{2}}{3}\left(\frac{f_{\phi}^{2}}{M_{\phi}}\right)
$$

where $e_{Q}=1 / 3$ is the charge factor of the $s$ quark in terms of the charge of electron.

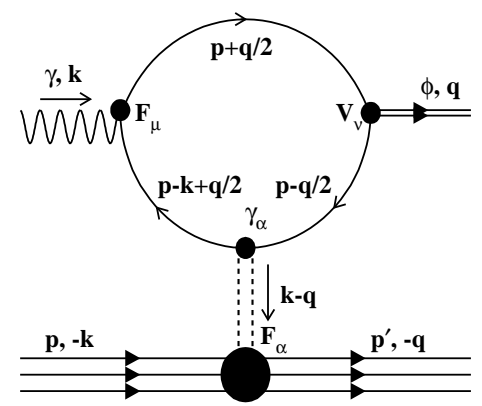

Fig. 1. Pomeron exchange diagram in the $\phi$ meson photoproduction.

Therefore, the current matrix element can be written as:

$$
\left\langle p_{f} m_{f}, q \lambda_{\phi}\left|J^{\mu}\right| p_{i} m_{i}\right\rangle=2 \beta_{0} t^{\mu \alpha \nu}(k, q) \epsilon_{\phi \nu}(q) \mathcal{G}_{\mathcal{P}}(s, t) \bar{u}\left(p_{f}\right) F_{\alpha}(t) u\left(p_{i}\right)
$$

where $u\left(p_{i}\right)$ and $\bar{u}\left(p_{f}\right)$ are the initial and final state Dirac spinors of the protons with four-momenta $p_{i}$ and $p_{f}$, respectively. $k$ and $q$ are the four-momenta of the incoming photon and outgoing $\phi$ meson, respectively. $\epsilon_{\phi \nu}$ is the polarization vector of the produced $\phi$ meson, and the factor 2 counts the equivalent contributions from Pomeron-quark and Pomeron-anti-quark interactions. $\mathcal{G}_{\mathcal{P}}(s, t)$ is related to the Regge trajectory of the Pomeron and has the form:

$$
\mathcal{G}_{\mathcal{P}}(s, t)=-i\left(\alpha^{\prime} s\right)^{\alpha(t)-1}
$$


where $\alpha(t)=1+\epsilon+\alpha^{\prime} t$ is the Regge trajectory of the Pomeron. The form factor $\mu_{0}^{2} /\left(\mu_{0}^{2}+p^{2}\right)$ is introduced for each off-shell quark line with four-momentum $p$. In Eq. (11), $t^{\mu \alpha \nu}(k, q)$ represents the loop tensor and it has the following expression for the contributing terms:

$$
t^{\mu \alpha \nu}(k, q)=(k+q)^{\alpha} g^{\mu \nu}-2 k^{\nu} g^{\alpha \mu} .
$$

Note that we have used the free constituent quark propagator $S(p)=-i /(\gamma$. $p+m_{s}$ ) for the strange quark with mass $m_{s}$. However, this latter mass term vanishes due to the odd number of $\gamma$ matrices in the trace for the loop. To preserve gauge invariance, we have adopted the transformation given in Ref. [11]. In section 4, we detail this point to investigate the effects from the different Pomeron exchange amplitudes due to the various schemes for fixing the gauge.

The above discussion shows that such a Pomeron exchange picture is consistent with the processes described by the effective Lagrangian in Eq. (1). Qualitatively, we suppose that the effective Lagrangian governs the coupling of the vector meson to the constituent $s$ and $\bar{s}$ in the Pomeron exchange. Then, the tensor part vanishes because odd number of $\gamma$-matrix appears in the loop integration. Only the vector part couples to the point-like vector meson effectively with the coupling constant $a$. This result reproduces the "on-shell approximation" for the $s \bar{s}$ - $\phi$ vertex. With this analogy, the parameter $a$ in the effective Lagrangian can be expressed as:

$$
a=f_{\phi} / M_{\phi}
$$

¿From Eq. (10), we have $f_{\phi}^{2} / M_{\phi}=26.6 \mathrm{MeV}$, then the value of parameter $a=0.16$ is derived. This value is comparable with what we use for the $s$ - and $u$-channel quark- $\phi$-meson coupling. However, such an analogy does not imply a rigorous constraint on the parameter $a$ since generally, the tensor coupling will contribute especially in the large $|t|$ region in the $s$ - and $u$-channel. But it provides us with a consistency test between the Pomeron exchange picture and the $s$ - and $u$-channel non-diffractive description. As discussed in the next section, with the constraint from the differential cross section, $|a|=0.15$ and $\left|b^{\prime}\right|=0.3$ give reasonable estimation of the contributions from the effective Lagrangian.

In the case of real photons, that is $Q^{2}=0$, the explicit expressions for the Pomeron exchange for the transverse and longitudinal $\phi$ production are

$$
\begin{aligned}
H_{a \lambda_{V}}= & 32 \sqrt{2} \alpha_{e} \pi \beta_{0}^{2} m_{\phi} f_{\phi} \\
& \times \frac{\mu_{0}^{2} F_{1}(t)}{\left(M_{\phi}^{2}-t\right)\left(2 \mu_{0}^{2}+M_{\phi}^{2}-t\right)}\left(\alpha^{\prime} s\right)^{\alpha(t)-1}\left[M_{T}(\mathcal{P})+M_{L}(\mathcal{P})\right]_{a \lambda_{V}},
\end{aligned}
$$


where

$$
\begin{aligned}
M_{T}(\mathcal{P})= & -\left[\left(\omega_{\gamma}+\omega_{\phi}\right)+\frac{\mathbf{q}^{2}}{E_{f}+M_{N}}+\frac{\mathbf{k}^{2}}{E_{i}+M_{N}}\right. \\
& \left.+\left(\frac{1}{E_{f}+M_{N}}+\frac{1}{E_{i}+M_{N}}\right) \mathbf{q} \cdot \mathbf{k}\right] \boldsymbol{\epsilon}_{\gamma} \cdot \boldsymbol{\epsilon}_{\phi} \\
& +\left(\frac{1}{E_{f}+M_{N}}+\frac{1}{E_{i}+M_{N}}\right) i \boldsymbol{\sigma} \cdot(\mathbf{k} \times \mathbf{q}) \boldsymbol{\epsilon}_{\gamma} \cdot \boldsymbol{\epsilon}_{\phi} \\
& +\frac{2}{E_{f}+M_{N}} \boldsymbol{\epsilon}_{\phi} \cdot \mathbf{k} \boldsymbol{\epsilon}_{\gamma} \cdot \mathbf{q} \\
& -\frac{2}{E_{f}+M_{N}} i \boldsymbol{\sigma} \cdot\left(\boldsymbol{\epsilon}_{\gamma} \times \mathbf{q}\right) \mathbf{k} \cdot \boldsymbol{\epsilon}_{\phi} \\
& +\frac{2}{E_{i}+M_{N}} i \boldsymbol{\sigma} \cdot\left(\boldsymbol{\epsilon}_{\gamma} \times \mathbf{k}\right) \mathbf{k} \cdot \boldsymbol{\epsilon}_{\phi}
\end{aligned}
$$

and

$$
\begin{aligned}
M_{\phi}|\mathbf{q}| \times M_{L}(\mathcal{P})= & -\omega_{\phi}\left(\omega_{\gamma}+\omega_{\phi}\right)-\omega_{\phi}\left(\frac{\mathbf{q}^{2}}{E_{f}+M_{N}}+\frac{\mathbf{k}^{2}}{E_{i}+M_{N}}\right) \\
& \left.-2 \frac{\omega_{\gamma}|\mathbf{q}|^{2}}{E_{f}+M_{N}}+\omega_{\phi}\left(\frac{1}{E_{f}+M_{N}}-\frac{1}{E_{i}+M_{N}}\right) \mathbf{q} \cdot \mathbf{k}\right] \mathbf{q} \cdot \boldsymbol{\epsilon}_{\gamma} \\
& -\left(\omega_{\gamma}|\mathbf{q}|^{2}-\omega_{\phi} \mathbf{q} \cdot \mathbf{k}\right) \frac{2}{E_{i}+M_{N}} i \boldsymbol{\sigma} \cdot\left(\boldsymbol{\epsilon}_{\gamma} \times \mathbf{k}\right) \\
& +\left(\omega_{\gamma}|\mathbf{q}|^{2}-\omega_{\phi} \mathbf{q} \cdot \mathbf{k}\right) \frac{2}{E_{f}+M_{N}} i \boldsymbol{\sigma} \cdot\left(\boldsymbol{\epsilon}_{\gamma} \times \mathbf{q}\right) \\
& +\omega_{\phi}\left(\frac{1}{E_{f}+M_{N}}+\frac{1}{E_{i}+M_{N}}\right) i \boldsymbol{\sigma} \cdot(\mathbf{k} \times \mathbf{q}) \mathbf{q} \cdot \boldsymbol{\epsilon}_{\gamma} .
\end{aligned}
$$

The subscripts $a(=1,2,3,4)$ and $\lambda_{V}(=0, \pm 1)$ denote the helicity elements of the amplitude in the helicity space.

It turns out to be impossible to extrapolate the theory of Pomeron exchange from high energy regions $\left(E_{\gamma}>>10 \mathrm{GeV}\right)$ down to the regions $3.0<E_{\gamma}<6.7$ $\mathrm{GeV}[23]$, with the same normalization factor $\beta_{0}$. The cross sections will be over-estimated if the same normalization factor is used in the low energy region. Thus, another normalization factor must be taken for the quark-Pomeron coupling $\beta_{s}$ at the nucleon-Pomeron vertex. We derive the normalization factor by fitting the data from Ref. [23] at $E_{\gamma}=6.45 \mathrm{GeV}$. It gives the $s$-quarkPomeron coupling strength $\beta_{s}=1.27 \mathrm{GeV}^{-1}$ (for instance, in Ref.[5] $\beta_{s}=1.5$ $\mathrm{GeV}^{-1}$ has been adopted). Qualitatively, the change of the normalization factor can be explained by the non-perturbative dressing of the quark-gluon vertex required by the Slavnov-Taylor identity, which introduces a nontrivial flavor dependence at the vertex [5]. In other words, we have made the assumption 
that the contribution from the flavor dependence of the quark-Pomeron vertex can be absorbed into the constant $\beta_{s}\left(=\beta_{0}\right)[2,5]$. Concerning the other parameters, we adopt the same values as used in Ref. [5]: $\epsilon=0.08, \alpha^{\prime}=0.25$ $\mathrm{GeV}^{-2}, \mu_{0}=1.2 \mathrm{GeV}$.

\section{3 t-channel $\pi^{0}$ exchange}

The Lagrangian for the $\pi^{0}$ exchange has the following form:

$$
L_{\pi N N}=-i g_{\pi N N} \bar{\psi} \gamma_{5}(\boldsymbol{\tau} \cdot \boldsymbol{\pi}) \psi
$$

for the $\pi N N$ coupling vertex, and

$$
L_{\phi \pi^{0} \gamma}=e_{N} \frac{g_{\phi \pi \gamma}}{M_{\phi}} \epsilon_{\alpha \beta \gamma \delta} \partial^{\alpha} A^{\beta} \partial^{\gamma} \phi^{\delta} \pi^{0}
$$

for the $\phi \pi \gamma$ coupling vertex, where the $\phi^{\delta}$ and $\pi^{0}$ represent the $\phi$ and $\pi^{0}$ fields, respectively, $A^{\beta}$ denotes the electromagnetic field, $\epsilon_{\alpha \beta \gamma \delta}$ is the LeviCivita tensor, and $M_{\phi}=1.02 \mathrm{GeV}$ is the mass of the $\phi$ meson. The $g_{\pi N N}$ and $g_{\phi \pi \gamma}$ in Eqs. (18) and (19) denote the coupling constants at the two vertices, respectively. Therefore, the transition amplitudes of the $t$-channel $\pi^{0}$ exchange have the following expression:

$$
\begin{aligned}
M_{T}\left(\pi^{0}\right)= & \frac{e_{N} g_{\pi N N} g_{\phi \pi \gamma}}{2 M_{\phi}\left(t-m_{\pi}^{2}\right)}\left[\omega_{\gamma} \boldsymbol{\epsilon}_{\gamma} \cdot\left(\mathbf{q} \times \boldsymbol{\epsilon}_{\phi}\right)+\omega_{\phi} \mathbf{k} \cdot\left(\boldsymbol{\epsilon}_{\gamma} \times \boldsymbol{\epsilon}_{\phi}\right)\right] \\
& \boldsymbol{\sigma} \cdot \mathbf{A} e^{-\frac{(\mathbf{q}-\mathbf{k})^{2}}{6 \alpha_{\pi}^{2}}}
\end{aligned}
$$

for the transverse transition, and

$$
M_{L}\left(\pi^{0}\right)=-\frac{e_{N} g_{\pi N N} g_{\phi \pi \gamma}}{2 M_{\phi}\left(t-m_{\pi}^{2}\right)} \frac{M_{\phi}}{|\mathbf{q}|}\left(\boldsymbol{\epsilon}_{\gamma} \times \mathbf{k}\right) \cdot \mathbf{q} \boldsymbol{\sigma} \cdot \mathbf{A} e^{-\frac{(\mathbf{q}-\mathbf{k})^{2}}{6 \alpha_{\pi}^{2}}}
$$

for the longitudinal transition, where $\omega_{\gamma}$ in the transition amplitudes denotes the energy of the photon with momentum $\mathbf{k}$, and the vector $\mathbf{A}=-\frac{\mathbf{q}}{E_{f}+M_{N}}+$ $\frac{\mathbf{k}}{E_{i}+M_{N}}$. The momentum transfer squared is $t=(q-k)^{2}=M_{\phi}^{2}-2 k \cdot q$. The factor $e^{-\frac{(\mathbf{q}-\mathbf{k})^{2}}{6 \alpha_{\pi}^{2}}}$ in Eqs. (20) and (21) plays a role like the form factor for both $\pi N N$ and $\phi \gamma \pi$ vertices. It comes out naturally in the harmonic oscillator basis since the nucleon is treated as a 3-quark system which is non-pointlike. Therefore, the expansion of the internal motion gives such a momentumdependent factor. The constant $\alpha_{\pi}$ in this form factor is treated as a parameter. 
Since $\alpha_{\pi}$ describes the combined form factor for both $\pi N N$ and $\phi \gamma \pi$ vertices, we do not expect it has the same value as $\alpha=410 \mathrm{MeV}$ which only corresponds to the $\phi N N$ vertex in the $s$ - or $u$-channel. We adopt the following values for the couplings:

$$
g_{\pi N N}^{2} / 4 \pi=14, g_{\phi \pi \gamma}^{2}=0.143, \alpha_{\pi}=300 \mathrm{MeV}
$$

It is worth noting that the inclusion of the $t$-channel $\pi^{0}$ exchange might result in a double-counting problem due to duality arguments. However, in the following sections, one can see that the $\pi^{0}$ exchange plays a quite negligible role in the $\phi$ meson photoproduction, which suggests that the duality hypothesis gives little constraint on this process. Since the duality problem is beyond a phenomenological study, we present results with or without the $\pi^{0}$ exchange to illustrate the effects in the following studies. 


\section{Observables and discussion}

In this work, we limit the discussion to the low energy region where the effects from nucleon resonances are expected to play a role in the reaction mechanism of the $\gamma p \rightarrow \phi p$ process.

\subsection{Cross-section}

As discussed in subsection 2.2, the picture of quark- $\phi$-meson couplings is consistent with each other in the Pomeron exchange and in the $s$ - and $u$-channel mechanisms. The analogy between the above two vertices leads to the value $a=0.16$. However, this approach does not put any constraint on the parameter $b^{\prime}(\equiv b-a)$, which contributes in the large $|t|$ region in the $s$ - and $u$-channel.

We have attempted to extract the values of the $a$ and $b^{\prime}$ parameters by fitting the differential cross section data. The result is :

$$
a=-0.035 \pm 0.166 ; b^{\prime}=-0.338 \pm 0.075
$$

First, let us mention that this result should be taken with caution since we fit the data at forward angles whereas the influence of $a$ and $b^{\prime}$ is at large angles. However, this result shows that parameter $b^{\prime}$ is well constrained while the constraint on parameter $a$ is loose. For $a$, rather than a precise value, the result provides a range which is consistent with the value reported in Section 2.2. In the $s$ - and $u$-channel, the parameter $a$ reflects the vector coupling of $\phi-u u$ or $\phi$ - $d d$ which should be suppressed by the OZI rule. In the above fitting, the small central value of $|a|=0.035$ shows some hints from such a suppression. However, recalling that one of our motivations is to investigate the sensitivities of the polarization observables to the small $s$ - and $u$-channel contributions, we have to take into account the large uncertainty in parameter $a$. In other words, larger value for $a$ is to give the upper limit of the sensitivities of the polarization observables. For $b^{\prime}$, the result favors a negative sign. Also, with the same motivation, we will present in the following the results for all the phase sets for $a$ and $b^{\prime}$ to provide a complete and systematic understanding of the role played by the $s$ - and $u$-channel contributions.

We hence fix the absolute values of the two parameters at the following values compatible with the above ranges:

$$
|a|=0.15,\left|b^{\prime}\right|=0.3
$$


As we will see below, these values allow to reproduce well enough the existing data on the cross section as well as the density matrix elements.

Notice that:

- The extreme value $|a|=0.15$ with different signs will show the maximum sensitivities of various observables to the resonance contributions. The OZI suppression considerations are discussed in section 3.5.

- Since we always have the combination of the parameters $b-a$ in the amplitudes, we define $b^{\prime} \equiv b-a$, and use $a$ and $b^{\prime}$ as the two parameters for the quark-vector-meson coupling.

- Once the signs of the two parameters $a$ and $b^{\prime}$ are determined, the phases between the $s^{-}, u$-channel amplitudes, Pomeron, and $\pi^{0}$ exchanges can be fixed.

- The above values, Eq. (22), are smaller than those estimated in Ref. [14]. The reason is that no Pomeron exchange was included there, therefore, the contributions from the non-diffractive effective Lagrangian were overestimated.

In Fig. 2, the results of our calculations for the differential cross section at $E_{\gamma}=2.0 \mathrm{GeV}$ are shown. The $\pi^{0}$-exchange contribution is small and limited to low momentum transfers. The $s$ - and $u$ - channel resonances produce an almost

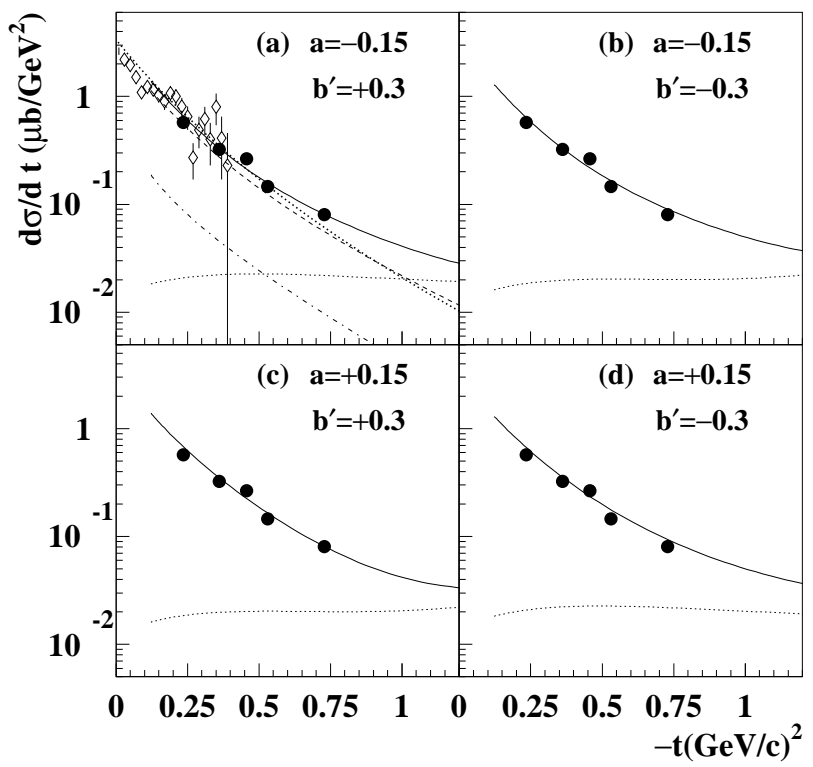

Fig. 2. Differential cross section at $E_{\gamma}=2.0 \mathrm{GeV}$ as a function of momentum transfer $(-t)$ for $\gamma p \rightarrow \phi p$. Data at $E_{\gamma}=2.0 \mathrm{GeV}$ (full circle) are from Ref. [24], and at $E_{\gamma}$ $=6.45 \mathrm{GeV}$ (diamond) from Ref. [23]. The curves are: i) $\pi^{0}$-exchange (dot-dashed); ii) $s$ - and $u$-channel contribution (dotted); iii) Pomeron exchange(dashed), and iv) contributions from i) to iii) (full curves). The Pomeron exchange at $E_{\gamma}=6.45 \mathrm{GeV}$ is also depicted (heavy dotted curve in (a)). Contributions from the Pomeron and $\pi^{0}$ exchanges (independent of $a$ and $b^{\prime}$ ) are only shown in (a). 
flat contribution which becomes dominant roughly above $|t|=1$. $(\mathrm{GeV} / \mathrm{c})^{2}$. For the Pomeron exchange, we show results at two energies (2.0 and 6.45 $\mathrm{GeV})$. The Pomeron exchange is the dominant contribution for $-t \leq 0.5$ $(\mathrm{GeV} / \mathrm{c})^{2}$, and shows a soft dependence on the energy. As seen in Figs. 2(b)(d), the differential cross section shows basically no sensitivity to the signs of the couplings $a$ and $b^{\prime}$.

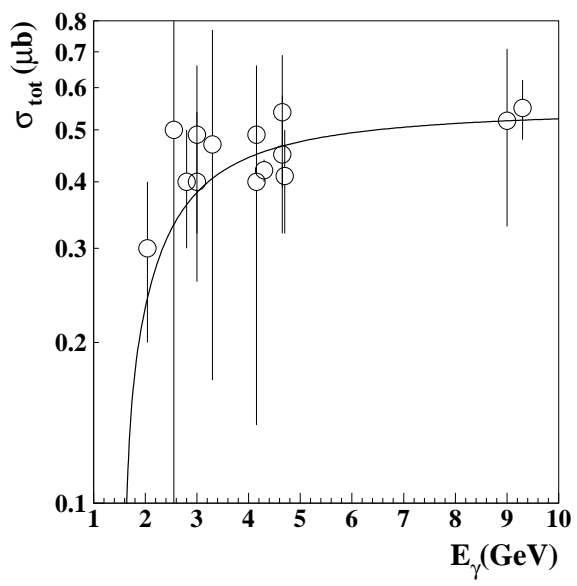

Fig. 3. The total cross section for $\gamma p \rightarrow \phi p$. The curve is the prediction of our full calculation and the data are from Ref. [25].

We also present predictions for the total cross section (Fig. 3). In spite of large uncertainties in the data, the theory-experiment comparison shows that our model reproduces consistently the dominating diffractive contributions in the whole phase space spanned by the data. Moreover, the almost energyindependent behavior of the $\phi$ meson photoproduction process is reproduced correctly in the model. From these considerations, we conclude that our treatment of the diffractive contribution is realistic enough. As emphasized in Introduction, based on such a reliable treatment, we can now investigate the role of the non-diffractive $\phi$ meson production mechanism in the $\gamma p \rightarrow \phi p$ process.

In the case of differential and total cross sections, the non-diffractive effects turn out to be small. However, as we will see below, such small effects can be amplified in polarization observables. The rest of this section is devoted to the polarization observable asymmetries where the differential cross section enters in the denominator.

\subsection{Single polarization asymmetries}

The beam polarization asymmetry $\check{\Sigma}$ at $E_{\gamma}=2.0 \mathrm{GeV}$ is shown in Fig. 4 . Comparing the Pomeron exchange (dashed curve in Fig. 4 (a)) with the Pomeron 
plus $\pi^{0}$ exchange (dotted curve in Fig. 4 (a)), we find that the contribution from $\pi^{0}$ exchange is negligible. The $s$ - and $u$-channel contributions amplified by the Pomeron exchange, due to the interference terms, increase the magnitude of the observable by about a factor of 3 around $110^{\circ}$ and produces a sign change above $150^{\circ}$ (dot-dashed curve in Fig. 4 (a)). The three mechanisms together produce the full curves in Fig. 4 (a) and (b). We present the results for the four phase sets in Fig. 4(b) for comparison.

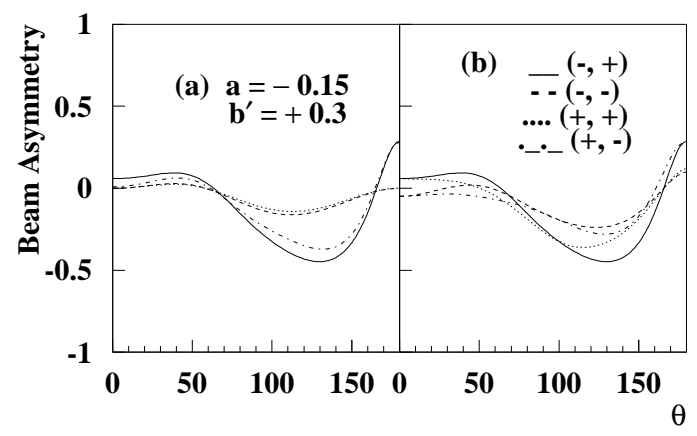

Fig. 4. The polarized beam asymmetry at $E_{\gamma}=2.0 \mathrm{GeV}$ with different phase signs. The curves in (a) stand for: Pomeron exchange (dashed), Pomeron and $\pi^{0}$ exchanges (dotted), Pomeron exchange and resonance contributions (dot-dashed), and the full calculation including all three components with $a=-0.15, b^{\prime}=0.3$ (full). In (b), the results of our full calculations for the four $\left(a, b^{\prime}\right)$ sets are depicted.

In Fig. 5 predictions for the target polarization asymmetry $\check{T} \equiv \mathbf{P}_{N} \cdot \hat{y} \mathcal{T}$, due to the same mechanisms discussed above in the case of the $\check{\Sigma}$ observable are reported.

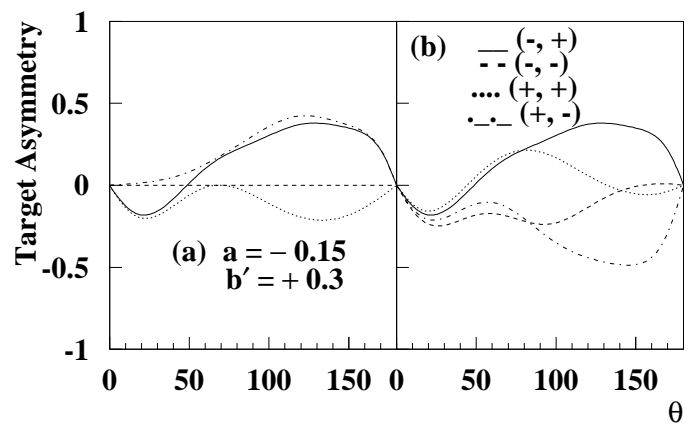

Fig. 5. Same as Fig. 4, but for the polarized target asymmetry.

Here, we wish to emphasize that the helicity amplitude structure of the $\check{\Sigma}$ observable differs drastically from those of the other single polarization observables. As summarized in Appendix, the $\check{\Sigma}$ observable is a bilinear combination of real-real or imaginary-imaginary parts, while the other three single polarization observables depend on real-imaginary couples. Moreover, the Pomeron exchange amplitude is treated purely imaginary in this model while that of 
the $\pi^{0}$ exchange is purely real. Therefore, the pure Pomeron exchange term, leads to a zero target asymmetry (dashed curve in Fig. 5(a)), while adding the $\pi^{0}$ exchange produces non-zero effects, especially at large angles (dotted curve in Fig. 5(a)). We find that a large cancelation arises between the longitudinal and transverse parts of the asymmetry, which produces a nearly zero asymmetry at $\sim 65^{\circ}$. This structure is independent on the relative phase between the Pomeron exchange and the $\pi^{0}$ exchange amplitudes, since the Pomeron exchange amplitude is purely imaginary and the $\pi^{0}$ exchange is purely real, therefore, the phase change will only give an overall sign to the dotted curve in Fig. 5(a).

The Pomeron plus resonances contributions (dot-dashed curve in Fig. 5(a)) gives even a larger asymmetry in magnitude, with opposite sign for backward angles, than the Pomeron plus $\pi^{0}$ exchange does. The full calculation (full curves in Fig. 5(a) and (b)) shows a minimum around $20^{\circ}$ due to $\pi^{0}$ exchange and a maximum around $130^{\circ}$ generated by the resonance terms. In both cases the Pomeron exchange plays an amplifying role in the predicted asymmetries. It shows that the target polarization asymmetry is governed mainly by the resonance contributions at large angles. For comparison, we also present the results with phase changes in Fig. 5(b).

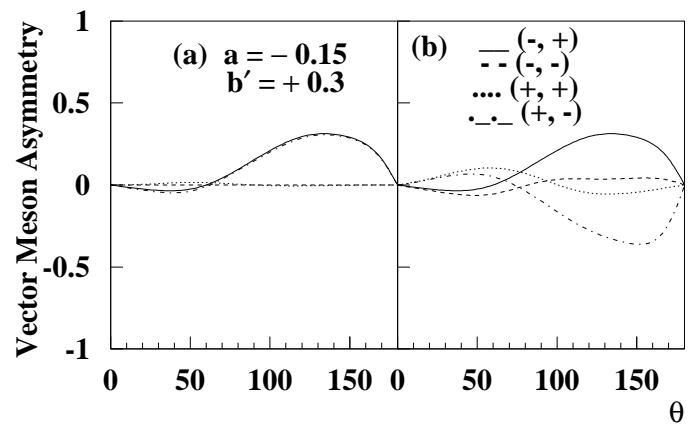

Fig. 6. Same as Fig. 4, but for the polarized vector meson asymmetry.

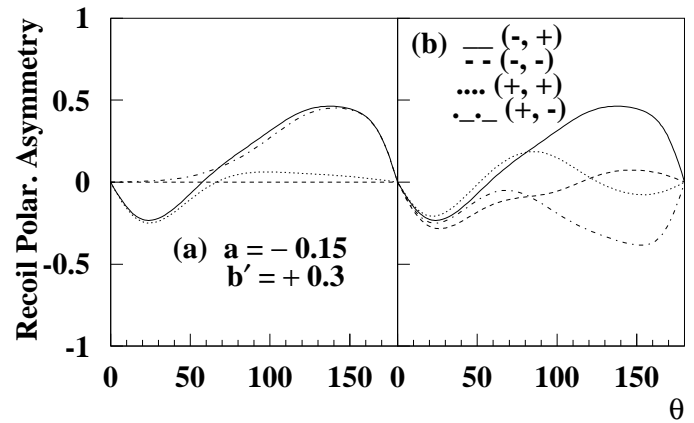

Fig. 7. Same as Fig. 4, but for the recoil polarization asymmetry. 
In Fig. 6, the vector polarization observable $\check{P}_{V}$ for the vector meson polarization is depicted. Here, the combined Pomeron and $\pi^{0}$ exchanges produce a very small positive asymmetry. The dominant effect is therefore due to the contributions from the $s$ - and $u$-channel resonances once again amplified by the Pomeron exchange (dot-dashed curve in Fig. 6(a)).

Finally, we show in Fig. 7 the recoil polarization asymmetry $\check{P}_{N^{\prime}}$.

To summarize the main features revealed by the single polarization observables:

- The Pomeron exchange mechanism turns out to be an efficient amplifier for the mechanisms suppressed in the cross sections.

- Although the influence of the $\pi^{0}$ exchange can be amplified in some polarization observables, it plays in general a rather minor role. Therefore, this might imply that the double-counting from duality (if it exits) is negligible.

- The nodal structure of the observables depends (in some cases heavily) on the signs of the two couplings $a$ and $b^{\prime}$.

- The $s$ - and $u$-channel resonances produce significant effects. The most favorable phase space region depends on the signs of the couplings $a$ and $b^{\prime}$ (Figs. 4(b), 5(b), 6(b), and 7(b)).

\subsection{Double polarization asymmetries}

Given the availability of polarized beam and polarized target, we now concentrate on the beam-target (BT) double polarization asymmetry. Another motivation in investigating this observable is that a recently developed strangeness knock-out model [11] suggests that a small $s \bar{s}$ component $(\sim 5 \%)$ in the proton might result in large asymmetries $(\sim 25-45 \%)$ in the BT observable at small angles. However, since the resonance contributions have not been taken into account there, an interesting question is: if contributions from the $s$ - and $u$-channel can produce a significant double polarization asymmetry without introducing strangeness component or not.

Our predictions are shown in Fig. 8. The Pomeron exchange alone (dashed curve in (a)), gives a negative asymmetry which increases in magnitude from forward to backward angles where the largest asymmetry is about $40 \%$. The $\pi^{0}$ exchange (dotted curve in (a)) diminishes slightly the asymmetry, while the resonances contributions (dot-dashed curve in (a)) enhances it. The full calculation leads finally to a decreasing behavior, going from almost zero at forward angles to $\sim-0.7$ at $180^{\circ}$. This result (full curve in Fig. 8(a) and (b)) is obtained with $a=-0.15$ and $b^{\prime}=+0.3$. The backward angle effects are also large in the case of $a=+0.15$ and $b^{\prime}=-0.3$ (dot-dashed curve in Fig. 8(b)). The situation becomes very different for the couplings sets with the 


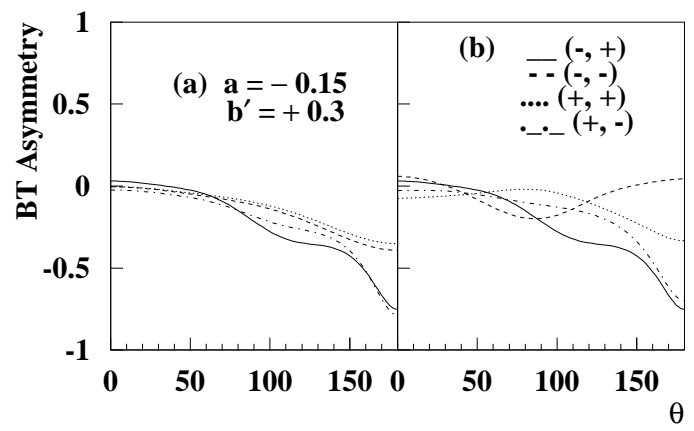

Fig. 8. Same as Fig. 4, but for the polarized beam-target asymmetry. The heavy dotted curve in (a) is given by the full calculation with $a=-0.015, b^{\prime}=+0.3$.

same signs: the effect is suppressed for $a=+0.15$ and $b^{\prime}=+0.3$ (dotted curve in Fig. 8(b)), and the shape changes drastically for $a=-0.15$ and $b^{\prime}=-0.3$ (dashed curve in Fig. 8(b)). The latter set produces (almost) vanishing values at extreme angles. The common feature to all four sets is that the beam-target asymmetry is small at forward angles.

\subsection{Density matrix elements}

In this subsection, the density matrix elements are investigated in the helicity system. Data for the density matrix elements at low energies [23] are still very sparse: measurements have been carried out only in the small $|t|$ region $\left(\theta_{c m}<20^{\circ}\right)$ at $E_{\gamma}=5.165$, and $6.195 \mathrm{GeV}$, where the Pomeron exchange dominates over other non-diffractive processes. In Fig. 9 the solid curve is the
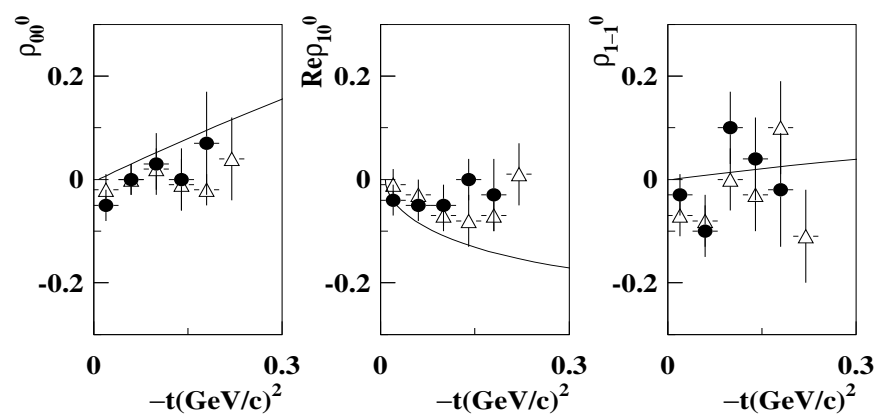

Fig. 9. Theoretical predictions for the density matrix elements at $E_{\gamma}=5.165 \mathrm{GeV}$. Data at $E_{\gamma}=5.165 \mathrm{GeV}$ (full circle) and at $E_{\gamma}=6.195 \mathrm{GeV}$ (triangle) are from Ref. [23]. $-t=0.3(\mathrm{GeV} / \mathrm{c})^{2}$ corresponds to $\theta_{c m} \sim 22^{\circ}$.

result of our calculation at $E_{\gamma}=5.165 \mathrm{GeV}$. At $E_{\gamma}=6.195 \mathrm{GeV}$, the results are not significantly different within this momentum transfer region, so, we do not show them here. Theory-data comparison shows again the character of 
diffractive dominance in the small $|t|$ region, and the Pomeron exchange (other amplitudes are included but negligible at this energy region) reproduces well enough the data for the three density matrix elements $\rho_{00}^{0}, \operatorname{Re} \rho_{10}^{0}$ and $\rho_{1-1}^{0}$.
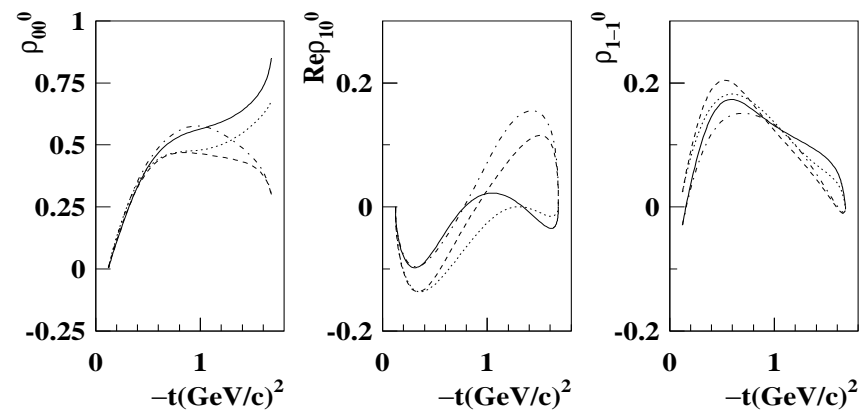

Fig. 10. Density matrix elements predicted at $E_{\gamma}=2.0 \mathrm{GeV}$ with phase changes for $\left(a, b^{\prime}\right)$ : solid $(-,+)$, dashed $(-,-)$, dotted $(+,-)$, and dot-dashed $(+,+)$ curves.
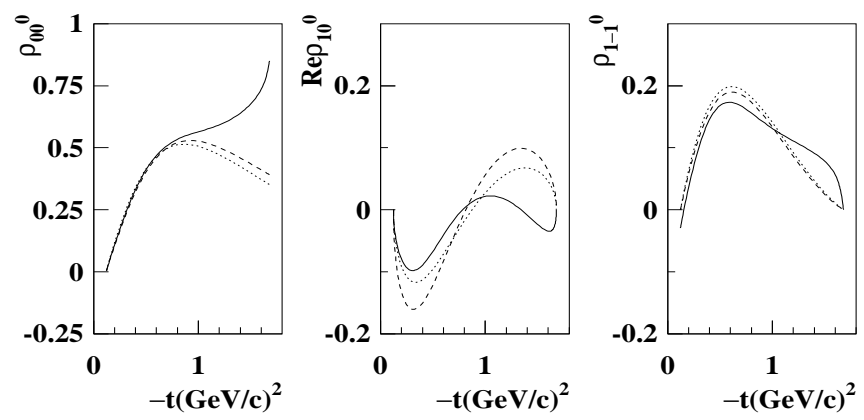

Fig. 11. Density matrix elements predicted at $E_{\gamma}=2.0 \mathrm{GeV}$ with $a=-0.15$, $b^{\prime}=0.3$. The curves stand for: pure Pomeron exchange (dashed), Pomeron plus $\pi^{0}$ exchanges (dotted), and full calculation (full).

In Fig. 10, our predictions for the three density matrix elements at $E_{\gamma}=2.0$ $\mathrm{GeV}$ are presented for different $a$ and $b^{\prime}$ phase sets. In Fig. 11, contributions from only the Pomeron exchange (dashed curve), Pomeron plus $\pi^{0}$ exchanges (dotted curve), and both exchanges plus the resonance contributions (full curve) are shown. The density matrix elements appear to be quite sensitive to the non-diffractive $s$ - and $u$-channel contributions. Also, the phase changes produce significant effects in the large $|t|$ region. However, in the forward direction, the matrix elements are not sensitive neither to the non-diffractive resonance effects nor to the phase changes. 


\subsection{OZI suppression effects}

As mentioned in subsection 3.1, the numerical results discussed there are obtained for $|a|=0.15$, which corresponds to no OZI suppression. Here, we report on the manifestations of such a suppression in the absence of OZI evading mechanisms. The OZI rule would imply a parameter value smaller by roughly one order of magnitude. To single out possible manifestations of the OZI suppression, we compare the numerical results for different observables for $|a|=0.15$ and $a=0.015$.

As already discussed, from the Pomeron-photon analogy picture, no constraint can be imposed on the other free parameter, $b^{\prime}$. However, the value $\left|b^{\prime}\right|=0.3$ could indicate an OZI suppression: the value extracted here is about one order of magnitude smaller than $b^{\prime}=2.5$ used in the $\omega$ and $\rho$ mesons photoproduction [14].

In Fig. 12, our results for three single polarization (beam, target, and vector meson), as well as the beam-target double polarization asymmetries are depicted. The polarized beam asymmetry shows little sensitivity to the phase of $a$, but depends significantly on the absolute value of this parameter. This observable is hence very appealing to study the OZI suppression effects and/or the related evading mechanisms. In the target and vector meson polarization asymmetries, the $a$-dependent terms come in basically in the interference terms and are mostly sensitive to the phase of this parameter. The beam-target double polarization asymmetry, turns out to result from cancellations among the helicity amplitudes, except at extreme backward angles where strong dependences on both the phase and magnitude of $a$ show up. This explains the large asymmetries (solid curve in Fig. 12) found for a small value of the coupling $a$. The curves without $s$ - and $u$-channel contributions (i.e. only Pomeron plus $\pi^{0}$ exchange) are also shown for comparison.

Density matrix elements are shown in Fig. 13 . The $\rho_{00}^{0}$ above $|t| \approx 1$. $(\mathrm{GeV} / \mathrm{c})^{2}$ shows a possibility to determine both the phase and the size of the $a$ parameter, while the $R e \rho_{10}^{0}$ depends strongly on the phase. The $\rho_{1-1}^{0}$ turns out to be dominated by the non-resonant terms (see Fig. 11).

The results presented here show clear sensitivity of some of the observables to the nucleon resonance contributions even in the presence of the OZI suppression mechanism. 


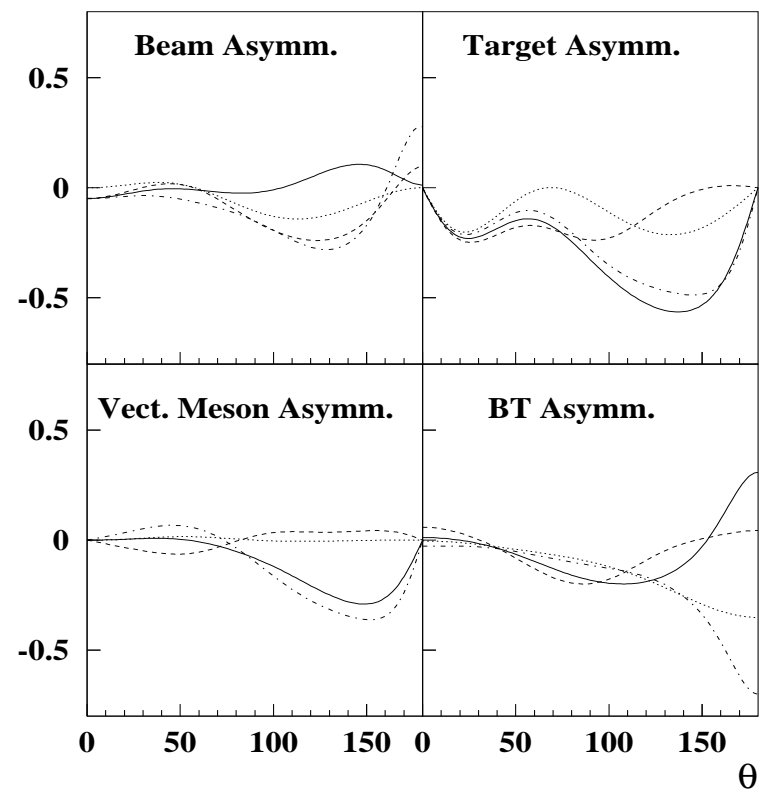

Fig. 12. Single and double polarization observables predicted at $E_{\gamma}=2.0 \mathrm{GeV}$ with $b^{\prime}=-0.3$. The curves stand for: Pomeron plus $\pi^{0}$ exchanges (dotted), and full calculation for $a=0.15$ (dot-dashed), $a=-0.15$ (dashed), and $a=0.015$ (solid).
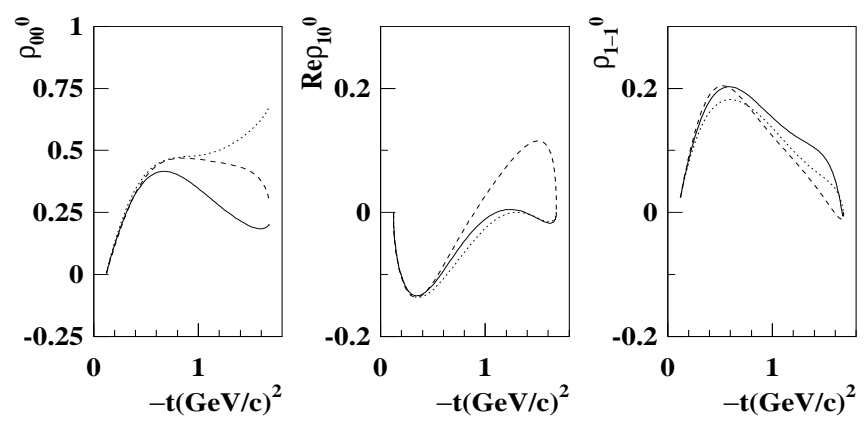

Fig. 13. Density matrix elements predicted at $E_{\gamma}=2.0 \mathrm{GeV}$ with $b^{\prime}=-0.3$. The curves stand for: full calculation for $a=0.15$ (dotted), $a=-0.15$ (dashed), and $a=0.015$ (solid). 


\section{Pomeron gauge invariance effects}

This section is devoted to investigate the sensitivity of the Pomeron exchange model to the various gauge-fixing schemes. This question arises from the fact that the Pomeron exchange is introduced phenomenologically to account for the diffractive behavior at small angles. However, at large angles, the description of the Pomeron structure is still an unsolved question. Here, we limit our discussion to the effects due to the different gauge-fixing schemes and the non-gauge-invariant one.

In Fig.1, the loop tensor is derived by supposing that the constituent quarks carry half of the momentum of the vector meson:

$$
\begin{aligned}
t^{\mu \alpha \nu}(k, q)= & 2 k^{\alpha} g^{\mu \nu}-\frac{2}{q^{2}} k^{\alpha} q^{\mu} q^{\nu}-2 g^{\alpha \mu}\left(k^{\nu}-q^{\nu} \frac{k \cdot q}{q^{2}}\right) \\
& +2\left(k^{\mu}-q^{\mu}\right)\left(g^{\nu \alpha}-\frac{q^{\alpha} q^{\nu}}{q^{2}}\right),
\end{aligned}
$$

where the second line violates gauge invariance. Taking into account that at the photon and vector meson vertices, $k \cdot \epsilon_{\gamma}=0$ and $q \cdot \epsilon_{\phi}=0$, one finds that the contributing terms are:

$$
t^{\mu \alpha \nu}(k, q)=2 k^{\alpha} g^{\mu \nu}-2 g^{\alpha \mu} k^{\nu}-2 q^{\mu} g^{\nu \alpha} .
$$

We note the above non-gauge-invariant Pomeron amplitude as t0. In Ref.[11], two schemes for restoring gauge invariance are employed. We refer reader to Ref.[11] for detailed discussion of how to derive the gauge-fixing terms, and skip to the final forms of the loop tensor.

In this work we have adopted the gauge-fixing scheme which gives the loop tensor as the following:

$$
\begin{aligned}
t^{\mu \alpha \nu}(k, q)= & (k+q)^{\alpha} g^{\mu \nu}-2 k^{\nu} g^{\alpha \mu} \\
& +2\left[k^{\mu} g^{\alpha \nu}+\frac{q^{\nu}}{q^{2}}\left(k \cdot q g^{\alpha \mu}-k^{\alpha} q^{\mu}-q^{\alpha} k^{\mu}\right)\right. \\
& \left.-\frac{k^{2} q^{\mu}}{q^{2} k \cdot q}\left(q^{2} g^{\alpha \nu}-q^{\alpha} q^{\nu}\right)\right]+(k-q)^{\alpha} g^{\mu \nu} .
\end{aligned}
$$

The contributing terms can be easily derived and are given by Eq. (13). Here we name this gauge-fixing scheme as $\mathbf{t} 1$.

Another gauge fixing scheme gives the contributing terms as:

$$
t^{\mu \alpha \nu}(k, q)=(k+q)^{\alpha} g^{\mu \nu}-k^{\nu} g^{\alpha \mu}-q^{\mu} g^{\alpha \nu},
$$


where those terms which do not contribute are omitted for brevity. We note this gauge-fixing scheme as $\mathbf{t} \mathbf{2}$.

Comparing these three kinds of Pomeron exchanges: t0, non-gauge-invariant; $\mathbf{t 1}$ and t2, gauge-invariant, one finds that these different Pomeron exchanges will produce different behaviors. However, one can identify the two terms which are different between the three Pomeron amplitudes: $q \cdot \epsilon_{\gamma} \gamma \cdot \epsilon_{\phi}$ and $q \cdot \gamma \epsilon_{\gamma}$. $\epsilon_{\phi}$, and finds that they generally play a role as a higher order contribution at forward angles. For $q \cdot \epsilon_{\gamma} \gamma \cdot \epsilon_{\phi}$ we write explicitly the non-relativistic expansion as follows:

$$
\begin{aligned}
M_{T}= & -\mathbf{q} \cdot \boldsymbol{\epsilon}_{\gamma} \frac{\mathbf{k} \cdot \boldsymbol{\epsilon}_{\phi}}{E_{i}+M_{N}} \\
& +\mathbf{q} \cdot \boldsymbol{\epsilon}_{\gamma}\left[\frac{i \boldsymbol{\sigma} \cdot\left(\boldsymbol{\epsilon}_{\phi} \times \mathbf{q}\right)}{E_{f}+M_{N}}-\frac{i \boldsymbol{\sigma} \cdot\left(\boldsymbol{\epsilon}_{\phi} \times \mathbf{k}\right)}{E_{i}+M_{N}}\right],
\end{aligned}
$$

and

$$
\begin{aligned}
M_{\phi}|\mathbf{q}| \times M_{L}= & -\mathbf{q} \cdot \boldsymbol{\epsilon}_{\gamma}\left(|\mathbf{q}|^{2}+\omega_{\phi}\left(\frac{\mathbf{q}^{2}}{E_{f}+M_{N}}+\frac{\mathbf{q} \cdot \mathbf{k}}{E_{i}+M_{N}}\right)\right) \\
& -\omega_{\phi} \mathbf{q} \cdot \boldsymbol{\epsilon}_{\gamma} \frac{i \boldsymbol{\sigma} \cdot(\mathbf{q} \times \mathbf{k})}{E_{i}+M_{N}}
\end{aligned}
$$

where $M_{T}$ and $M_{L}$ represent the transverse and longitudinal transition amplitude, respectively. Also, this term is the one which makes the difference between the two gauge-fixing schemes, t1 and t2. However, at forward angles, this term becomes small and vanishes since $\mathbf{q}$ has almost the same direction as $\mathbf{k}$ in the forward scattering, therefore, the product $\mathbf{q} \cdot \boldsymbol{\epsilon}_{\gamma}$ becomes very small. Qualitatively, we can see that the product of $\mathbf{q} \cdot \boldsymbol{\epsilon}_{\gamma}$ gives an overall suppression of this term, which guarantees the consistent behavior of the Pomeron exchanges at small angles with different gauge-fixing schemes.

For the second term, $q \cdot \gamma \epsilon_{\gamma} \cdot \epsilon_{\phi}$, which is also essential to restore gauge invariance, we find that there is also an overall suppression of the longitudinal amplitude from $\mathbf{q} \cdot \boldsymbol{\epsilon}_{\gamma}$ in the forward direction:

$$
\begin{aligned}
M_{\phi}|\mathbf{q}| \times M_{L}= & -\mathbf{q} \cdot \boldsymbol{\epsilon}_{\gamma}\left(\omega_{\phi}^{2}+\omega_{\phi}\left(\frac{\mathbf{q}^{2}}{E_{f}+M_{N}}+\frac{\mathbf{q} \cdot \mathbf{k}}{E_{i}+M_{N}}\right)\right) \\
& -\omega_{\phi} \mathbf{q} \cdot \boldsymbol{\epsilon}_{\gamma} \frac{i \boldsymbol{\sigma} \cdot(\mathbf{q} \times \mathbf{k})}{E_{i}+M_{N}}
\end{aligned}
$$

Note that in Eqs. (28) and (29), except for the first term in the first line, the other terms are identical. Therefore, the substitution of Eq. (28) and 
(29) into scheme $\mathbf{t} \mathbf{2}$ in the longitudinal amplitude for the two terms gives, $M_{L}=-M_{\phi} \mathbf{q} \cdot \boldsymbol{\epsilon}_{\gamma} /|\mathbf{q}|$, which is obviously suppressed in the forward direction.

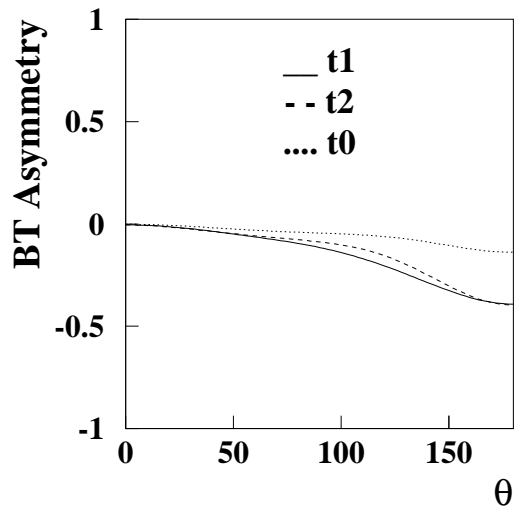

Fig. 14. Beam-target asymmetry for different Pomeron exchange amplitudes. The non-diffractive contributions have been switched off.

For the transverse amplitude of the $q \cdot \gamma \epsilon_{\gamma} \cdot \epsilon_{\phi}$ term, the explicit expression is:

$$
\begin{aligned}
M_{T}= & -\left(\omega_{\phi}+\frac{\mathbf{q}^{2}}{E_{f}+M_{N}}+\frac{\mathbf{q} \cdot \mathbf{k}}{E_{i}+M_{N}}\right) \boldsymbol{\epsilon}_{\gamma} \cdot \boldsymbol{\epsilon}_{\phi} \\
& -\frac{i \boldsymbol{\sigma} \cdot(\mathbf{q} \times \mathbf{k})}{E_{i}+M_{N}} \boldsymbol{\epsilon}_{\gamma} \cdot \boldsymbol{\epsilon}_{\phi}
\end{aligned}
$$

where the second term is suppressed by $(\mathbf{q} \times \mathbf{k})$ in the forward direction. However, for the first term in the above equation, the only suppression comes from the relatively smaller momentum of the massive $\phi$ meson.

In Fig.14, the beam-target asymmetry for different Pomeron exchanges (with only the different Pomeron contribution) are presented. It shows that they consistently converge to small asymmetry at forward angles while at large angles the asymmetry is quite sensitive to the particular Pomeron structure. The solid curve is given by the $\mathbf{t} \mathbf{1}$ gauge-fixing scheme and the dotted curve by t2. The non-gauge-invariant Pomeron to (dashed curve) has a similar behavior as $\mathbf{t} 1$. Therefore, a question arising from this result is that "if the nondiffractive contributions are taken into account, the polarization asymmetries are sensitive to the Pomeron structure or not?" Since the Pomeron structure at large angles is unknown, a gauge-dependent Pomeron interference at large angles might make the asymmetry predictions misleading. To investigate this aspect, we calculate the polarization asymmetries with different Pomeron exchanges given by t0, t1 and t2, and the results are presented in Fig. 15 and 16. Comparing the results given by the different Pomeron exchanges, we find 


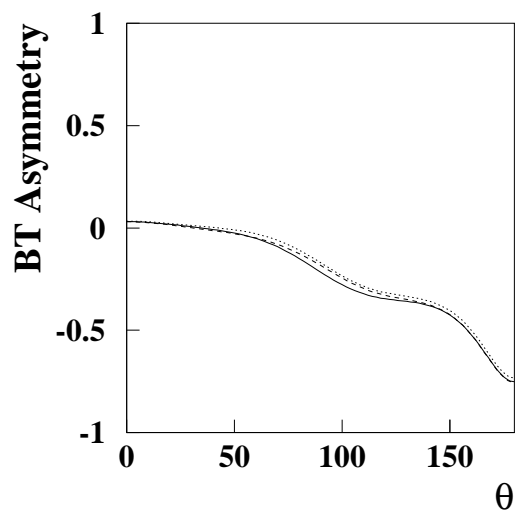

Fig. 15. Beam-target asymmetries with all the contributions taken into account. The Pomeron exchange is given by $\mathbf{t} \mathbf{1}$ (full), t2 (dashed), and to (dotted). $a=-0.15$, $b^{\prime}=0.3$ have been adopted for the $s$ - and $u$-channel contributions.

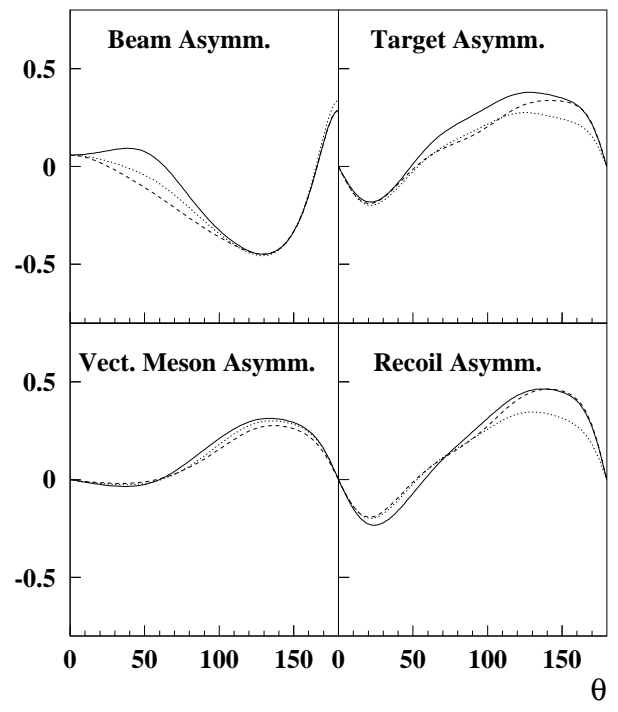

Fig. 16. Single asymmetries with all the contributions taken into account. The curves have the same meanings as in Fig. 15.

that they have very similar behaviors in the polarization asymmetry observables which shows that the asymmetries are not sensitive to the Pomeron structures when the non-diffractive contributions are taken into account. In other words, although different gauge-fixing schemes are introduced for the Pomeron exchange terms, they produce a quite gauge-independent interference in the polarization asymmetries, which suggests that the asymmetries can be considered mainly determined by the non-diffractive contributions. Since the asymmetries predicted at large angles have little dependence on the Pomeron exchange models, the experimental observation can provide a direct 
test of the model for the $s$ - and $u$-channel reactions.

In summary, although the Pomeron exchange can have different structures due to the different gauge-fixing schemes, it does not influence significantly the polarization observables at large angles. Therefore, the main feature of an asymmetry can be regarded as depending more on the non-diffractive contributions rather than on the particular Pomeron structure. 


\section{Conclusions}

In this paper we have investigated the reaction $\gamma p \rightarrow \phi p$ in the region close to threshold $\left(E_{\gamma}^{\text {thres }} \sim 1.57 \mathrm{GeV}\right)$. The known dominant diffractive process is taken into account via the t-channel Pomeron exchange. The main novelty of our work is the study of the role played by the $s$ - and $u$-channel intermediate nucleonic resonances. This is done using a quark-model based effective Lagrangian approach. Our formalism includes also the small non-diffractive contribution from the $t$-channel $\pi^{0}$ exchange.

In the first step, the nucleonic resonance effects are investigated in the $S U(6) \otimes$ $O(3)$ symmetry limit. In the polarization observables, the small nucleonic resonance contributions produce significant asymmetries at large angles, however, the asymmetries are quite small at forward angles.

Based on the different gauge-fixing schemes for the Pomeron exchange terms, three kinds of Pomeron amplitudes are investigated. It shows that these amplitudes converge to a similar behavior at small angles, but have quite different behaviors at large angles when only the Pomeron exchange contributes. However, with the non-diffractive $s$ - and $u$-channel contribution taken into account, we find that the asymmetries at large angles are governed by the non-diffractive contributions. Conclusively, the asymmetries are not sensitive to the different Pomeron exchange amplitudes due to gauge invariance. This gives us the confidence that at large angles the ambiguity arising from the Pomeron structure can be neglected in this model.

The quark model presented in this work is based on the $S U(6) \otimes O(3)$ symmetry. However, the breaking of this symmetry will lead to the configuration mixings among states with the same quantum numbers. This mechanism allows those resonances excluded by virtue of the $S U(6) \otimes O(3)$ symmetry to contribute in this reaction and suppresses the role played by other members of the degenerate states. The present data on the $\phi$ meson photoproduction are too scarce to allow a close study of the possible deviations from the exact $S U(6) \otimes O(3)$ symmetry. As a rough estimation of such an effect, we studied the role of individual resonances by including or eliminating their contributions in the polarization observables. We find that most of the single and double polarization observables investigated here show significant effects only at large angles.

In summary, the forward angle polarization asymmetries are almost insensitive to the $s$ - and $u$-channel nucleonic resonance contributions (as well as to the $t$-channel $\pi^{0}$ exchange). This is an interesting finding, since data at small angles might be able to shed a light on other sources, such as the small $s \bar{s}$ component in the nucleon. At large angles, significant sensitivities to the 
phases of the couplings provide insights to the small, but still sizable $s$ - and $u$-channel resonance contributions. These results hold also in the case of OZI suppression relevance. Some of the phase sets for those parameters $\left(a\right.$ and $\left.b^{\prime}\right)$, offer also a test of deviations from the exact $S U(6) \otimes O(3)$ symmetry.

Finally, we would like to point out a few possible weak points in this work, as summarized below:

i) The $s$ - and $u$-channel contributions are calculated within a quark model, while the Pomeron exchange model is based on the Regge phenomenology. So, the consistency between the two frames remains to be investigated.

ii) The form factor $F_{1}(t)$ for the Pomeron coupling to the proton has no relation with the exponential form factor in the quark model which comes naturally from the spatial integrals over the baryon wavefunctions for the resonance excitation and the $\pi^{0}$ exchange contributions.

Upcoming data from JLAB [26] are expected to allow to disentangle various components of the reaction mechanism investigated here. Confrontation between the present theoretical predictions and data might show the limits of the non-relativistic constituent quark approach presented here and the need for more sophisticated and a fully relativistic formalism.

\section{Acknowledgments}

One of us (Q. Z.) expresses his thanks to IPN-Orsay and the "Bourses de Recherche CNRS-K.C. WONG" for financial support. Two of us (Q. Z. and B. S.) would like to express appreciations to Zhenping Li for his interest in this work and much encouragement. Q. Z. acknowledges the beneficial discussions with C. Bennhold and W. Kloet. 


\section{Appendix}

Generally, the helicity amplitudes can be explicitly written as:

$$
H_{a \lambda_{V}} \equiv H_{a \lambda_{V}}^{r}+i H_{a \lambda_{V}}^{i}
$$

where $H_{a \lambda_{V}}^{r}$ and $H_{a \lambda_{V}}^{i}$ represent the real and imaginary part of the amplitude, respectively. $\lambda_{V}(=0, \pm 1)$ is the helicity of the vector meson. With the $\Gamma$ and $\omega$ matrices given by Ref. [19], the four single and one double polarization asymmetries of the spin observables can be expressed explicitly as follows:

Polarized photon asymmetry

$$
\begin{aligned}
\check{\Sigma}= & \frac{1}{2}\left\langle H\left|\Gamma^{4} \omega^{A}\right| H\right\rangle \\
= & \frac{1}{2}\left\{-H_{1-1}^{r} H_{41}^{r}-H_{1-1}^{i} H_{41}^{i}+H_{10}^{r} H_{40}^{r}+H_{10}^{i} H_{40}^{i}\right. \\
& -H_{11}^{r} H_{4-1}^{r}-H_{11}^{i} H_{4-1}^{i}+H_{2-1}^{r} H_{31}^{r}+H_{2-1}^{i} H_{31}^{i} \\
& \left.-H_{20}^{r} H_{30}^{r}-H_{20}^{i} H_{30}^{i}+H_{21}^{r} H_{3-1}^{r}+H_{21}^{i} H_{3-1}^{i}\right\} .
\end{aligned}
$$

Polarized target asymmetry

$$
\begin{aligned}
\check{T} & =-\frac{1}{2}\left\langle H\left|\Gamma^{10} \omega^{1}\right| H\right\rangle \\
& =\sum_{\lambda_{V}}\left\{H_{1 \lambda_{V}}^{r} H_{2 \lambda_{V}}^{i}-H_{1 \lambda_{V}}^{i} H_{2 \lambda_{V}}^{r}+H_{3 \lambda_{V}}^{r} H_{4 \lambda_{V}}^{i}-H_{3 \lambda_{V}}^{i} H_{4 \lambda_{V}}^{r}\right\} .
\end{aligned}
$$

Polarized vector meson asymmetry

$$
\begin{aligned}
\check{P}_{V}= & \frac{1}{2}\left\langle H\left|\Gamma^{1} \omega^{3}\right| H\right\rangle \\
= & \frac{\sqrt{3}}{2}\left\{H_{1-1}^{r} H_{10}^{i}-H_{1-1}^{i} H_{10}^{r}+H_{2-1}^{r} H_{20}^{i}-H_{2-1}^{i} H_{20}^{r}\right. \\
& \left.H_{3-1}^{r} H_{30}^{i}-H_{3-1}^{i} H_{30}^{r}+H_{4-1}^{r} H_{40}^{i}-H_{4-1}^{i} H_{40}^{r}\right\} .
\end{aligned}
$$

Recoil polarization asymmetry

$$
\begin{aligned}
\check{P}_{N^{\prime}} & =\frac{1}{2}\left\langle H\left|\Gamma^{12} \omega^{1}\right| H\right\rangle \\
& =\sum_{\lambda_{V}}\left\{H_{3 \lambda_{V}}^{i} H_{1 \lambda_{V}}^{r}-H_{3 \lambda_{V}}^{r} H_{1 \lambda_{V}}^{i}+H_{4 \lambda_{V}}^{i} H_{2 \lambda_{V}}^{r}-H_{4 \lambda_{V}}^{r} H_{2 \lambda_{V}}^{i}\right\} .
\end{aligned}
$$


The explicit expression for the component $\mathcal{C}_{z z}^{\gamma N}$ in the beam-target double polarization asymmetry is given by:

$$
\begin{aligned}
\mathcal{C}_{z z}^{\gamma N}= & \frac{1}{2}\left\langle H\left|\Gamma^{9} \omega^{1}\right| H\right\rangle \\
= & \frac{1}{2} \sum_{\lambda_{V}}\left\{H_{1 \lambda_{V}}^{r} H_{1 \lambda_{V}}^{r}+H_{1 \lambda_{V}}^{i} H_{1 \lambda_{V}}^{i}-H_{2 \lambda_{V}}^{r} H_{2 \lambda_{V}}^{r}-H_{2 \lambda_{V}}^{i} H_{2 \lambda_{V}}^{i}\right. \\
& \left.+H_{3 \lambda_{V}}^{r} H_{3 \lambda_{V}}^{r}+H_{3 \lambda_{V}}^{i} H_{3 \lambda_{V}}^{i}-H_{4 \lambda_{V}}^{r} H_{4 \lambda_{V}}^{r}-H_{4 \lambda_{V}}^{i} H_{4 \lambda_{V}}^{i}\right\}
\end{aligned}
$$

It is worth noting that the helicity amplitudes can be explicitly related to the density matrices which can be measured in experiments [20]. 


\section{References}

[1] T.H. Bauer, R.D. Spital, D.R. Yennie and F.M. Pipkin, Rev. Mod. Phys. 50, 261 (1978).

[2] A. Donnachie and P.V. Landshoff, Phys. Lett. B 185, 403 (1987); Nucl. Phys. B 311, 509 (1989).

[3] J.-M. Laget and R. Mendez-Galain, Nucl. Phys. A 581, 397 (1995).

[4] L.P.A. Hackmann, A. Kaidalov and J.H. Koch, Phys. Lett. B 365, 411 (1996).

[5] M.A. Pichowsky and T.-S.H. Lee, Phys. Lett. B 379, 1 (1996); Phys. Rev. D 56, 1644 (1997).

[6] R.A. Williams, Phys. Rev. C 57, 223 (1998).

[7] S. Okubo, Phys. Lett. 5, 165 (1963);

G. Zweig, CERN Report TH-412 (1964);

I. Iizuka, Prog. Theor. Phys. Suppl. 37, 21 (1966).

[8] J. Reifenrother et al., ASTERIX Collaboration, Phys. Lett. B 267, 299 (1991);

M.A. Faessler et al., Crystal Barrel Collaboration, Phys. At. Nucl. 57, 1693 (1994);

V.G. Ableev et al., OBELIX Collaboration, ibid, 57, 1716 (1994); Phys. Lett. B 334, 237 (1994).

[9] E.M. Henley, G. Krein, S.J. Pollock and A.G. Williams, Phys. Lett. B 269, 31 (1991);

E.M. Henley, G. Krein and A.G. Williams, ibid B 281, 178 (1992).

[10] J. Ellis, M. Karliner, D.E. Kharzeev and M.G. Sapozhnikov, Phys. Lett. B 353, 319 (1995).

[11] A.I. Titov, Y. Oh and S.N. Yang, Phys. Rev. Lett. 79, 1634 (1997);

A.I. Titov, Y. Oh, S.N. Yang and T. Morii, Phys. Rev. C 58, 2429 (1998).

[12] A. Titov, T.-S. H. Lee and H. Toki, nucl-th/9812074.

[13] Q. Zhao, Z.P. Li and C. Bennhold, Phys. Lett. B 436, 42 (1998).

[14] Q. Zhao, Z.P. Li and C. Bennhold, Phys. Rev. C 58, 2393 (1998).

[15] S. Capstick and N. Isgur, Phys. Rev. D 34, 2809 (1986).

[16] Zhenping Li, Phys. Rev. D 52, 4961 (1995);

Zhenping Li, Hongxing Ye, and Minghui Lu, Phys. Rev. C 56, 1099 (1997).

[17] Z.P. Li and B. Saghai, Nucl. Phys. A 644, 345 (1998).

[18] R.G. Moorhouse, Phys. Rev. Lett. 16, 772 (1966).

[19] M. Pichowsky, Ç. Şavklı and F. Tabakin, Phys. Rev. C 53, 593 (1996). 
[20] K. Schilling, P. Seyboth and G. Wolf, Nucl. Phys. B 15, 397 (1970).

[21] Particle Data Group, C. Caso et al., Euro. Phys. J. C 3, 1 (1998).

[22] Zhenping Li, Phys. Rev. D 48, 3070 (1993).

[23] H.-J. Behrend et al., Nucl. Phys. B 144, 22 (1978).

[24] H.J. Besch et al., Nucl. Phys. B 70, 257 (1974).

[25] H.R. Crouch et al., Phys. Rev. 156, 1426 (1967);

R. Erbe et al., Phys. Lett. B 27, 54 (1968);

R. Erbe et al., Phys. Rev. 175, 1669 (1968);

M. Davier, Phys. Rev. D 1, 790 (1969);

J. Ballam et al., Phys. Rev. D 7, 3150 (1973);

D.P. Barber et al., Z. Phys. C 12, 1 (1982).

[26] E.S. Smith et al., JLAB proposal E93-022;

C. Marchand et al., JLAB proposal E93-031;

D.J. Tedeschi et al., JLAB proposals E97-005 and E-98-109. 